\title{
Delivery and Diffusion of Retinal in Dermis and Epidermis Through The Combination of Prodrug Nanoparticles and Detachable Dissolvable Microneedles
}

\section{Pattrawadee Toprangkobsin}

Chulalongkorn University

Wijit Banlunara

Chulalongkorn University

Benchaphorn Limcharoen

Chulalongkorn University

Asada Leelahavanichkul

Chulalongkorn University

Pravit Asawanonda

Chulalongkorn University

Chanat Kumtornrut

Chulalongkorn University

Titiporn Sansureerungsikul

Mineed Technology, Thailand Science Park

Teeranut Rutwaree

Mineed Technology, Thailand Science Park

Supason Wanichwecharungruang ( $\nabla$ psupason@chula.ac.th )

Chulalongkorn University https://orcid.org/0000-0002-2802-4341

\section{Research Article}

Keywords: Retinaldehyde, Microneedles, Stability, Dermis, Drug release

Posted Date: November 9th, 2021

DOl: https://doi.org/10.21203/rs.3.rs-642577/v1

License: (c) (1) This work is licensed under a Creative Commons Attribution 4.0 International License. Read Full License 
Version of Record: A version of this preprint was published at Drug Delivery and Translational Research on February 21st, 2022. See the published version at https://doi.org/10.1007/s13346-022-01136-3. 


\section{Abstract}

To minimize fast chemical degradation of retinal, we convert this aldehyde into proretinal nanoparticles (PRNs) by forming retinylidene moieties on chitosan and allowing the grafted polymers to assemble into nanoparticles, and then load the obtained PRNs into detachable microneedles made of 1:1 (by weight) hyaluronic acid/maltose. An embedment of the PRNs in the solid matrix of microneedles helps improving chemical stability of the grafted retinal; the loaded device can be kept at $25^{\circ} \mathrm{C}$ for three months (longest time experimented) with less than $30 \%$ degradation of the retinylidene moieties. The presence PRNs in the hyaluronic acid-maltose matrix also help improving mechanical strength of the needles. Administration of PRN-loaded detachable microneedles on fresh porcine ear skin results in complete deposition of an array of microneedles in the skin tissue at the dept that spans both epidermis and dermis, as observed by stereomicroscopic and confocal fluorescence microscopic analyses of the crosssectioned tissue pieces. Obvious diffusion of the PRNs from the originally embedded site of the needles in the skin tissue to the nearby location can be observed, and even distribution in the tissue is reached at $4 \mathrm{~h}$ post administration. Rats administered with single dose of PRN-loaded microneedles show significant increased epidermal thickness as compared to rats administered with unloaded microneedles. Both PRNloaded microneedles and unloaded microneedles produce no skin irritation in rats.

\section{Introduction}

Retinoids are group of compounds that include vitamin A or retinoic acid and its derivatives such as retinol, retinal, retinyl palmitate, retinyl acetate and others. Retinoids are important modulators of immune response and essential regulators for cell proliferation, differentiation, and morphogenesis during skin development [1-3]. At the epidermis layer, retinoids regulate epidermal keratinocyte growth and differentiation [4, 5]. At dermal layer, they not only stimulate growth and differentiation of fibroblast and endothelial cells but also regulate various functions of these cells resulting in the reduction in matrix metalloproteinase and the increase in newly synthesized collagenous matrix in dermal tissue [6]. These functions make retinoid an effective anti-aging compound that can exert its anti-aging effect in both dermis and epidermis [7]. Besides anti-aging, retinoid has been used in the treatment of numerous dermatological conditions, including acne [8], psoriasis [9], ichthyosis [10], and actinic keratosis [11].

Nevertheless, skin applications of retinoid face problem, especially on skin irritation $[12,13]$. Skin irritation from retinoids occurs at the epidermis through the induction of excess keratinocyte, causing epidermal slough [12-14]. In addition, production of interleukin-1 in the growth-stimulated keratinocytes is responsible for proinflammatory effects [6]. These effects in epidermis result in skin erythema, exfoliation, dryness, burning, scaling, and even alopecia [15]. Because skin irritation of retinoid is dose dependent [16], lower topical dose of retinoid is used to minimize this adverse effect. This thwarts the use of high concentration flux of topical retinoid to push the compound into the dermis for more effective anti-aging function at dermal fibroblast. Research has shown that topical application of vitamin A can provide only around $5 \%$ bioavalbility to skin tissue, and most of the $5 \%$ accumulate in the epidermis [17, 18]. 
Another problem in the topical use of retinoids is their chemical instability in cosmetic and pharmaceutical formulations. A report on long-term and accelerated stability testing of retinoids in various liquid and cream-based products revealed retinoid instabilities in almost all products, with upto $80 \%$ and $100 \%$ degradation after 6 months when kept at $25^{\circ} \mathrm{C}$ and $40^{\circ} \mathrm{C}$, respectively. Stability is formulation-dependent, wherein not dependent on the retinoid concentration but dependent on the type of retinoid derivative [19]. Encapsulation of retinoid into various particles have been shown to help improving stability of retinoid [20-23]. Nevertheless, these carriers could not help delivering retinoid to dermis.

Dissolvable microneedles (DMNs) consist of an array of micron-sized needles made from biocompatible materials that can dissolve in skin tissue. These 100-1000 $\mu \mathrm{m}$ hight needles can be fabricated with drugs inside the needles and safely embedded into skin tissue [24, 25]. Upon the dissolution of the embedded needles, drugs are released to viable epidermis and dermis [26-28]. A recent report has confirmed a long-term safety of repeating uses of DMNs in human volunteers [29]. By embedding retinoid into DMNs, its stability can be improved [30]. It was reported that DMNs could deliver retinoid into dermis more effectively than conventional topical formulations [31]. The use of retinoid-loaded DMNs for the treatment of seborrheic keratosis or senile lentigo has also been demonstrated [32,33].

Here we demonstrate the combined use of prodrug nanoparticles and detachable DMNs to both stabilize and deliver retinoid into skin tissue. The study includes the optimization of needle materials, the effect that the prodrug nanoparticles produce on the physical characteristic of the DMNs, the distribution of the retinoid in the DMNs, and the chemical stability of the retinoid when being directly loaded into DMNs and when being prepared into prodrug nanoparticles and then loaded into DMNs. The work also covers ex vivo experiments that show dissolution rate of the microneedles in the skin tissue and the diffusion of the retinoid delivered by DMNs in the tissue. Lastly, in vivo effects of proretinal nanoparticle-loaded DMNs on irritation and epidermal thickness of rat skin are also reported.

\section{Materials And Methods}

\section{Preparation of pro-retinal nanoparticles (PRNs)}

PRNs were prepared by grafting retinal (Sigma-Aldrich) onto chitosan (CS, Mw of 40,000-50,000 Da, Taming Enterprise, Samut-Sakhon, Thailand) polymer. The grafting was carried out using our previously reported protocol [34]. In brief, CS was dissolved in $0.1 \%$ acetic acid, and the $\mathrm{pH}$ of the obtained solution was adjusted to 5.9 using $\mathrm{NaOH}$. The final solution contained $45 \mathrm{mg}$ CS in $19.0 \mathrm{~mL}$ solution. The grafting reaction was initiated by slowly adding retinal $(15 \mathrm{mg}$ in $1.0 \mathrm{~mL}$ of ethanol) dropwise into the two-neck round-bottom flask containing aqueous $\mathrm{CS}$ suspension at $5^{\circ} \mathrm{C}$, under ultrasonic ( $40 \mathrm{kHz}$ ), light-proof and $\mathrm{N}_{2}$ atmospheric condition. After stirring for $2 \mathrm{~h}$, the $\mathrm{pH}$ of the solution was adjusted to 6.8 , and PRN suspension was obtained. The particles size of PRN was examined by scanning electron microscopic (SEM, JSM-6400; JEOL, Tokyo, Japan).

\section{Composite materials for microneedles}


Binary mixtures of various polymers and sugar were experimented for microneedle fabrication. Hyaluronic acid (HA, injection grade, Shandong Focuschem Biotech Co., Ltd., Shandong Sheng, China) was mixed with each of the following materials: polyvinyl pyrrolidone (PVP K30, average Mw 40,000, Sigma-Aldrich, Germany), polyvinyl alcohol (PVA, average Mw 146,000-186,000, Sigma-Aldrich), carboxymethyl cellulose (CMC, Dow Chemical Thailand Ltd, Bangkok, Thailand), sericin (Seritech Co., Ltd, Bangkok, Thailand) and maltose (Sigma-Aldrich), at two ratios. Solution of selected materials was prepared in water and the obtained solution was used to prepare microneedle arrays via the molding method as previously described $[31,35]$. The total of 11 formulations of needle matrix material were experimented and they include (expressed here as weight percentages) 100\% HA, 50\% PVP + 50\% HA, $25 \% \mathrm{PVP}+75 \% \mathrm{HA}, 50 \%$ maltose $+50 \% \mathrm{HA}, 25 \%$ maltose $+75 \% \mathrm{HA}, 50 \% \mathrm{PVA}+50 \% \mathrm{HA}, 25 \% \mathrm{PVA}+75 \%$ $\mathrm{HA}, 50 \% \mathrm{CMC}+50 \% \mathrm{HA}, 25 \% \mathrm{CMC}+75 \% \mathrm{HA}, 50 \%$ sericin $+50 \% \mathrm{HA}$ and $25 \%$ sericin $+75 \% \mathrm{HA}$. Microneedles used in mechanical testing and stability experiments were $8 \times 8 \mathrm{~mm}$ patch containing an array of $10 \times 10$ needles of the tetragonal pyramidal shape with $300 \times 300 \mu \mathrm{m}$ base, $650 \mu \mathrm{m}$ needle height, the tip-to-tip distance of $500 \mu \mathrm{m}$. The needles were sitting on a $500 \mu \mathrm{m}$ thick base made of the same material as the needles.

For DMN patches that contain PRN or retinal, the preparation was the same except that PRN or retinal was added to the polymer solution. PRN-loaded microneedles were prepared by dissolving HA (40 mg) and maltose ( $40 \mathrm{mg}$ ) in the PRN suspension ( $3 \mathrm{mg} \mathrm{CS}, 1 \mathrm{mg}$ retinal, $1 \mathrm{~mL}$ of $7 \%$ ethanol in water). The obtained mixture was loaded into the mold and let dry as described above. Retinal-loaded DMNs were prepared by dissolving HA (40 mg) and maltose $(40 \mathrm{mg})$ into retinal solution (1 mg retinal, $1 \mathrm{~mL} 7 \%$ ethanol in water) and the obtained mixture was loaded into the mold and let dry as previously described.

All the obtained microneedles were examined under stereomicroscope (Olympus DP22, Japan). Mechanical testing was carried out on DMN patches using the universal testing machine (UTM, Shimadzu EZ-S, Shimadzu Corporation, Tokyo, Japan). Distribution of retinal and PRNs in the DMN patches were acquired by taking confocal fluorescence images (Confocal fluorescence microscope, Eclipse, Ti series microscope, Nikon, Japan) of each DMN patch at all layers and then stacking the obtained images into three dimensional images.

\section{Detachable dissolvable microneedles for ex vivo and in vivo experiments}

PRN-loaded detachable dissolvable microneedle (DDMN) patch was prepared by dissolving HA (40 mg) and maltose ( $40 \mathrm{mg}$ ) in the PRN suspension ( $3 \mathrm{mg}$ CS, $1 \mathrm{mg}$ retinal, $1 \mathrm{~mL}$ of $7 \%$ ethanol in water). The obtained mixture of $0.05 \mathrm{ml}$ was loaded into the silicone mold, and the filled mold was put in the lightproof and moisture-controlled ( 5\%) chamber until dry. Then $0.05 \mathrm{ml}$ of vicious polymer solution (without PRN) was dropped onto the dry microneedle array (in the PDMS mold) and the water penetrable lint-free sheet $(600 \mu \mathrm{m}$ thick woven polyester sheet) was attached, then the assembled piece was put in the light-proof and moisture-controlled ( 2\%) chamber until the array was completely dry. Retinal-loaded DDMN patch was prepared similarly except that the freshly made retinal solution was used in placed of PRN suspension. Retinal solution was prepared by dissolving $1 \mathrm{mg}$ of retinal into $1 \mathrm{~mL}$ of $7 \%$ ethanol in 
water. The unloaded DDMN patch was prepared similarly except that water was used in placed of the PRN suspension. DDMN patches used in ex vivo experiment. The obtained DDMN patches were $8 \times 8 \mathrm{~mm}$ patch containing an array of $10 \times 10$ needles of the tetragonal pyramidal shape with $300 \times 300 \mu \mathrm{m}$ needle base, $650 \mu \mathrm{m}$ needle height, the tip-to-tip distance of $500 \mu \mathrm{m}$ and were used for ex vivo drug diffusion experiments. DDMN patches used for in vivo skin irritation experiment were prepared similarly with adjusted amount of PRN ( $8 \mu \mathrm{g}$ of retinal per patch), and the patch was $8 \times 8 \mathrm{~mm}$ patch containing an array of $10 \times 10$ needles of the tetragonal pyramidal shape with $100 \times 100 \mu \mathrm{m}$ base, $150 \mu \mathrm{m}$ needle height, the tip-to-tip distance of $500 \mu \mathrm{m}$.

\section{Retinal stability}

Chemical stability of retinylidene moieties in PRN-loaded DMNs (amount of retinal $=50 \mu \mathrm{g}$ per patch) and in PRN suspension (concentration of retinal $=1 \mathrm{mg} / \mathrm{mL}$ of the suspension), and stability of retinal in retinal-loaded DMNs (amount of retinal $=50 \mu \mathrm{g}$ per patch) and in retinal solution (concentration of retinal $=1 \mathrm{mg} / \mathrm{mL}$, solvent $=7 \%(\mathrm{v} / \mathrm{v})$ ethanol in water $)$, were examined.

Each DMN sample was kept in individual light-proof package at $4,25,40$, and $50{ }^{\circ} \mathrm{C}$. At each time point $(0,7,14,30,60$, and 90 days), the DMN sample was taken out and subjected to extraction and quantification. For retinal-loaded DMN or PRN-loaded DMN, the DMN patch was dissolved in $2 \mathrm{~mL}$ acidic water $(0.0025 \mathrm{M} \mathrm{HCl})$ under $\mathrm{N}_{2}$ atmosphere and light-proof condition. The solution was left for 10 min. Then the solution was partitioned with ethyl acetate ( 3 times with $2 \mathrm{~mL}$ ethyl acetate each time). The collected ethyl acetate extract was quantified for retinal by UV-vis spectrophotometry using maximum wavelength of $370 \mathrm{~nm}$ with the aid of a calibration curve.

For retinal solution or PRN suspension, the samples were kept under light-proof condition at the same temperatures and same durations. At each time point, the liquid sample was subjected to retinal extraction. The extraction was initiated by transferring $5 \mathrm{M} \mathrm{HCl}$ into $2 \mathrm{~mL}$ of the sample to reach the final $\mathrm{HCl}$ concentration of $0.0025 \mathrm{M}$. The mixture was left under $\mathrm{N}_{2}$ and light-proof condition for 10 min before subjecting to ethyl acetate partition (3 times with $2 \mathrm{~mL}$ ethyl acetate each time). The collected ethyl acetate extract was quantified for retinal as described above.

\section{Ex vivo drug diffusion in skin tissue}

Fresh porcine ear from local slaughterhouse (Lopburi, Thailand) was cleaned with citrate buffer ( $0.1 \mathrm{M}$, $\mathrm{pH}$ 7.4). The hairs were shaved, and the ear was rinsed with water and dried with tissue paper. The DDMN samples (650 $\mu \mathrm{m}$ of needles height) were pressed against the full-thickness porcine ear skin, then $1 \mathrm{drop}$ of water was applied and the DDMNs were pressed for $1 \mathrm{~min}$, then the backing sheet was removed. The ear was kept in a close petri dish lined with PBS buffer-soaked paper towel with the subcutaneous side touching the paper towel (to keep the tissue moist) for 0,1 and $4 \mathrm{~h}$. At each time point, the full thickness skin was surgically sectioned, and the tissue section was examined under stereomicroscope and confocal fluorescence microscope. Diffusion of retinoid in skin tissue pieces was examined under 
stereomicroscope and confocal fluorescence microscope (Eclipse, Ti series microscope, Nikon, Japan) using the excitation and emission wavelengths of $525 \mathrm{~nm}$ and $488 \mathrm{~nm}$, respectively.

\section{In vivo skin irritation}

The study protocol was approved by the committee of the use of animal for the scientific purpose of Chulalongkorn University animal care (protocol no. 1873021). Eight male rats (Wistar rat, 10-12 weeks, 300-400 g, Nomura Siam International Co. Ltd., Thailand) were housed at Chulalongkorn University Laboratory Animal Center, in an isolated clean room held at $25 \pm 2{ }^{\circ} \mathrm{C}$, with a relative humidity of $65-75 \%$. The rats were acclimatized for 3 weeks before starting the experiment. Rats were divided into two groups of 4 rats each: $24 \mathrm{~h}$ and $7 \mathrm{~d}$ groups.

For the treatment, rats were anesthetized and their hairs at the treated area were shaved. Each rat was treated with PRN-loaded DDMN patch (equivalent to $8 \mu \mathrm{g}$ retinal per patch) on the right dorsal skin, and unloaded DDMN patch on the left dorsal skin. The administration was carried out by pressing the DDMN patch against the skin using pressure from fingertips for $1 \mathrm{~min}$, then one drop of water was dropped over the backing of the DDMN patch, and the patch was hold for another $1 \mathrm{~min}$. After that, the base sheet of the DDMN patch was taken out. The treated area was observed at $1,6,12,24 \mathrm{~h}$ and every day until day 7 post the administration. Full thickness skin of the test area was sampled at $24 \mathrm{~h}$ and $7 \mathrm{~d}$ post the single administration. The biopsied skin tissue was fixed in $10 \%$ formalin buffer and processed as routine histopathological techniques. The skin sections were evaluated by the Draize scoring system. Erythema and edema were scored as follows: 0 = neither erythema nor edema; 1 = very slight erythema and/or barely perceptible edema; 2 = well-defined erythema and/or slight edema; $3=$ moderate to severe erythema or moderate edema, and $4=$ severe erythema and/or edema. Thickness of epidermis was measured on digital expanded images of the cross sectioned skin tissue pieces. The samples include 1) control group at $24 \mathrm{~h}$ post application (unloaded DDMN patch), 2) control group at 7 days post application (unloaded DDMN patch), 3) treated group at $24 \mathrm{~h}$ post application (PRN-loaded DDMN patch) and 4) treated group at 7 days post application (PRN-loaded DDMN patch). There were 12 application sites for each group (3 sites/animal, 4 animal/group). Three measurements were done for each application sites. The total of numbers of measurements were 36 measurements/group.

\section{Statistical Analysis}

Difference between epidermal thickness of rats from the unloaded-DDMN-treated group and the PRNloaded DDMN-treated group was analyzed by unpaired t-test through the GraphPad Prism version 9.1.1 (225) software (GraphPad, USA). Here the $p$ value of $<0.001$ was used to indicate significant difference.

\section{Results And Discussion}

\section{DMNs and mechanical property}


The DMNs patches of $8 \times 8 \mathrm{~mm}^{2}$ in the form of an array containing $10 \times 10$ square pyramidal needles (300×300 $\mu \mathrm{m}$ base, $650 \mu \mathrm{m}$ needle height, and tip-to-tip distance of $500 \mu \mathrm{m})$ could be successfully fabricated from either HA alone or from the binary composites of HA with each of the 5 different materials, PVP, PVA, CMC, sericin and maltose. Here the square pyramidal-shaped needle was selected over conical-shaped needle due to its superior skin penetration ability [36], and over trigonal pyramidal needle due to its higher active entrapment volume [37].

Among HA, PVP, PVA, CMC, sericin and maltose, HA is the only naturally abundant material in skin tissue and can be degraded in the body by hyaluronidase [38]. Therefore, for maximum compatibility to skin tissue, which is the delivery site of microneedles, HA should be the most desirable structural material of the needles. However, DMNs made of pure non-crosslinked HA showed large, displaced distance against compressive force (Fig. 1 A-1 and B-1, red line), indicating its low hardness, and agreeing well with the deformed needles after being pressed (Fig. 1 D-1). To improve the mechanical strength, we explored the binary composites of HA with the five materials, PVP, PVA, CMC, sericin and maltose. The second component was used at $25 \%$ and $50 \%(\mathrm{w} / \mathrm{w})$ of HA. Among the five materials mixed with HA, CMC showed no improvement of mechanical strength when added at either 25 or $50 \%$ (Fig. 1 A-1, A-2 and F-1). Significant improvement of rigidity could be achieved by adding PVA at $25 \%$ and $50 \%$, or sericin at $25 \%$, or PVP at $50 \%$, or maltose at $50 \%$. DMNs made of these composites showed less displaced distances when being pressed (Fig. 1).

Although adding sericin at $25 \%$ gave good mechanical property, we did not select this formulation for further study due to the obvious skin irritation observed for microneedles containing sericin (data not shown). The 1:1 maltose-HA and the 1:1 PVP-HA gave DMNs of comparable good mechanical properties, and the obtained needles could hold the structure against $50 \mathrm{~N}$ force (Fig. 1A-2). The 1:1 PVP-HA and the 1:1 maltose-HA were, therefore, used as DMN base material for the next experiments.

\section{Pro-retinal nanoparticles (PRN)-loaded microneedles}

Pro-retinal nanoparticles or PRNs were prepared as previously described by grafting retinal onto chitosan polymer via an imine linkage and allowing the retinylidene chitosan to self-assemble into water dispersible nanoparticles [34]. As expected, 200-300 nm yellowish particles that easily and stably disperse in water were obtained (Fig. 2A). The loading of retinal in the particles was $212 \mathrm{mg}$ of retinal/g of PRN particles.

The obtained PRN was loaded into the 1:1 PVP-HA-DMNs (DMNs with 50\% PVP and 50\% HA as needle matrix material) and the 1:1 maltose-HA-DMNs (DMNs with 50\% maltose and 50\% HA as needle matrix material). Unexpectedly, when the PRNs were mixed with PVP-HA polymer mixture, aggregation of PRNs took place immediately. As a result, only PRN-loaded 1:1 maltose-HA-DMN was fabricated (Fig. 2B1 D1). The loading content was $59 \mathrm{mg} P R N / g$ of the obtained DMNs. Each $0.36 \mathrm{~cm}^{2}$ DMN patch contained $0.24 \mathrm{mg}$ of PRN in the needles which corresponded to $50 \mu \mathrm{g}$ of retinal. In addition to the loading of PRNs into the DMNs, free retinal was also loaded into the DMNs at the same retinal loading (Fig. 2B2 - D2). PRN-loaded DMNs appeared yellowish and showed even distribution of PRNs in the DMNs when 
observed through stereomicroscope (Fig. 2B1 and C1). Well distribution of the free retinal in the DMNs could also be observed using the same technique (Fig. 2B2 and C2). Although the PRN-loaded DMN patch and the retinal-loaded DMN patch contained the same amount of retinal, the former gave stronger fluorescence at $\lambda_{\mathrm{ex}} / \lambda_{\mathrm{em}}$ of $488 / 525 \mathrm{~nm}$. We speculate that interactions between retinylidene moieties and chitosan polymer in the rigid self-assembled PRNs probably limit rotational and vibrational freedom of the retinylidene moieties. As a result, the non-radiative vibrational relaxation of the excited retinylidene moieties in these PRNs was less probable than that of free retinal molecules in the needle matrix. This automatically propelled more deactivation of the excited species via fluorescence pathway for the retinylidene in the PRNs, resulting in stronger fluorescence of the PRN in the DMN as compared to that of the free retinal in the DMN.

The PRN-loaded DMNs, free retinal-loaded DMNs and the unloaded DMNs (made of 1:1 HA/maltose) showed small difference in rigidity (Fig. 1A-3). The PRN-loaded DMNs, free retinal-loaded DMNs and the unloaded DMNs (made of 1:1 HA/maltose) could withstand the compressive force of 75, 60 and $55 \mathrm{~N}$, respectively (Fig. 1A-3). The two loaded ones could withstand a little more force as compared to the unloaded DMNs. This is not surprising because both PRN and retinal are immiscible with the watersoluble polymer matrix and could act as filler particles that help increasing mechanical strength of the needle polymer matrix.

\section{Stability of retinal}

We previously reported that the stability of retinal could be improved when the compound was converted into PRN [7]. Nevertheless, it was still not enough to be used in liquid formulations of consumer products for which long shelf-life is needed. Here we speculated that by embedding PRNs in solid microneedles, stability of the retinoid moieties should be further improved. Experimental result, indeed, showed great improvement in stability of the retinylidene moieties when kept in microneedles as compared to the unembedded PRNs and the free retinal (Fig. 3B, C and D). The improvement is likely the combined effects of 1) the retinylidene localization inside the rigid nanoparticles (self-assembled PRN) which limits access of oxidizing species to the retinoid moiety, 2) the embedment of the PRNs inside solid needle matrix which significantly slowdown chemical reaction when compared to liquid medium, and 3) the abundance of maltose, the compound with reducing property, in the needle matrix. These protection mechanisms are likely the foundation of good retinoid stability for the PRN-loaded DMNs kept at 4 and $25^{\circ} \mathrm{C}$. When comparing retinoid stability between the PRN-loaded DMNs versus the retinal-loaded DMNs (Fig. 3A and B), and PRN suspension versus retinal solution (Fig. $3 \mathrm{C}$ and D), it is obvious that the self-assembled PRN plays an important part in stability improvement. When comparing between PRN-loaded DMNs versus PRN suspension (Fig. 3A and C), the effect of solid matrix encompassing the PRN can be realized clearly.

\section{Ex vivo drug diffusion}

Next, we monitored the release of retinal in the skin tissue that was applied with the PRN-loaded DDMNs or the retinal-loaded DDMNs. Yellow color of retinal and retinylidene moieties was used to tract its 
whereabout in the cross-sectioned tissue pieces (Fig. 4A and B). The result showed clear embedment of arrays of microneedles in the skin tissues (Fig. 4A1 and B1). The shape and depth (distance from the stratum corneum side of the skin tissue) of the yellow color in the skin tissue roughly matched with the dimension of the applied microneedles $(300 \times 300 \mu \mathrm{m}$ square base with $650 \mu \mathrm{m}$ needle height). The embedment was at both epidermis and dermis layers. After $1 \mathrm{~h}$, the yellow color at the originally embedded sites could still be detected (Fig. 4A3 and B3). At $4 \mathrm{~h}$ post administration, obvious yellow color all over epidermis and dermis of the porcine skin tissue was observed (Fig. 4A4 and B4), indicating diffusion of the retinoid from the original deposited sites. Both retinal-loaded DDMNs and PRN-loaded DDMNs showed the retinoid diffusion in skin tissue.

Next, the experiment was repeated using fluorescence signal at $\lambda_{\mathrm{ex}} / \lambda_{\mathrm{em}}$ of $488 / 510 \mathrm{~nm}$ from the retinoid to locate its whereabout in the cross-sectioned porcine skin tissue. Here only skin administered with PRNloaded DDMNs showed detectable fluorescence signals. Very low intensity signal could be detected from tissue administered with retinal-loaded DDMNs (data not shown). This was not surprising because, as mentioned and explained in Sect. 3.2, free retinal, as compared to PRN, probably possesses higher nonradiative vibrational relaxation which results in less fluorescence emission. For the PRN-loaded DDMN administered porcine skin tissue, we observed the distribution of fluorescence signals at both 1 and $4 \mathrm{~h}$ post administration (Fig. 4C, D and E). With these obvious fluorescence signals, the presence of PRNs in both epidermis and dermis could be confirmed. The obvious spot-like high intensity fluorescence signals were observed and such characteristic implied that the signals were from PRNs and agglomerated PRNs. We speculate that even at $4 \mathrm{~h}$ post administration, not all the imine bonds linking retinal to chitosan in the PRNs were hydrolyzed. This was deliberated from the obvious spot-like high intensity fluorescence signals which implied that the signals were still from agglomerated particles. Some low intensity diffusive fluorescence signals could also be detected, and this likely originated from the released retinal molecules. This implied sustained release of retinoid from the deposited PRNs.

Both the stereomicroscopic and the fluorescence results indicate that DDMNs could deliver PRNs into epidermal and dermal skin layers and release of retinal from the deposited PRNs could take place slowly in skin tissue. Since skin irritation from retinoid usually occurs at epidermis [12-14], an ability to deliver retinoid into dermis without high accumulation in epidermis, may help tackle the skin irritation side effect frequently observed in topical retinoid application. As a result, we next test for skin irritation of the PRNloaded DDMNs on rats.

\section{Rat skin irritation}

Next the PRN-loaded DDMNs and the unloaded DDMNs were tested for skin irritation on dorsal skin of rats. No erythema, edema or other sign of irritation could be observed during the 7 days monitoring period (Fig. 5). At day 7 post single administration, the group applied with PRN-loaded DDMNs gave healthy skin with lighter color. Images of hematoxylin and eosin-stained cross sectioned histological skin also revealed healthy skin tissue in both groups. It should be noted here that the amounts of PRNs in each patch was equivalent to $8 \mu \mathrm{g}$ retinal per $0.64 \mathrm{~cm}^{2}$ patch which accounted for the administration dose of 
$12.5 \mu \mathrm{g}$ retinal per $\mathrm{cm}^{2}$. In normal topical retinoid formulation, applying $0.1 \mathrm{~mL}$ of $0.01 \%$ retinoid cream on area of $1 \mathrm{~cm}^{2}$ gives around $10 \mu \mathrm{g}$ retinoid per $\mathrm{cm}^{2}$.

Both the sustained release of retinal from PRNs and the distribution of the PRNs in dermis and epidermis (demonstrated in the above ex vivo experiment) probably help reducing the high accumulation of retinal in upper epidermis, therefore, no skin irritation was observed in all rats administered with PRN-loaded DDMNs. Both the unloaded DDMN (control) and the PRN-loaded DDMN did not produced any adverse reaction to rat skin. Epidermis of the group applied with PRN-loaded DDMNs showed significant increased thickness at day 7 as compared to the controlled group. The average epidermal thicknesses were $62.10 \pm 11.25 \mu \mathrm{m}$ and $43.38 \pm 9.65 \mu \mathrm{m}$ for the PRN-loaded DDMNs and the unloaded DDMNs, respectively (Fig. $5 \mathrm{C} 2$ and D2).

\section{Conclusion}

Experiments have indicated that the combination of HA:maltose at 1:1 produced a material with enough compressive strength to penetrate into the porcine and rat skin. The material could be fabricated into detachable dissolvable microneedles. Loading of proretinal nanoparticles or PRNs into the HA:maltose DDMNs further improved the mechanical property of the microneedles, i.e., microneedles loaded with PRNs could withstand higher compressive force as compare to the unloaded microneedles. Retinoid in the PRN-loaded microneedles showed less than 30\% and 20\% degradation when kept for three months at $25^{\circ} \mathrm{C}$ and $4{ }^{\circ} \mathrm{C}$, respectively. Ex vivo porcine skin administered with PRN-loaded microneedles showed PRNs distribution in both epidermis and dermis. No skin irritation was observed in rats administered with PRN-loaded microneedles.

\section{Declarations}

\section{Acknowledgments}

P.T. thanks the faculty of science, Phranakhon Si Ayutthaya Rajabhat University for granting her leave permission to pursue her doctoral studies. This work was funded by the National Research Council of Thailand (809/2563) and the Center of Excellence in Advanced Materials and Biointerfaces, Chulalongkorn University.

\section{Funding}

This work was funded by the National Research Council of Thailand (809/2563) and the Center of Excellence in Advanced Materials and Biointerfaces, Chulalongkorn University.

\section{Conflicts of interest/Competing interests}

The authors declare no competing interests.

\section{Availability of data and material}


Data available on request from the authors.

\section{Code availability}

Not applicable

\section{Authors' contributions}

P.T. designed the study, performed experiments, and wrote the manuscript. S.W. directed the research and assisted in writing and editing the manuscript. All the authors have provided comments on the manuscript.

\section{Ethics approval}

All the animal experiments were performed according to Chulalongkorn University animal care (CULAC); protocol no. 1873021.

\section{Consent to participate}

Not applicable

\section{Consent for publication}

Not applicable

\section{References}

1. Roche FC, Harris-Tryon TA. Illuminating the Role of Vitamin A in Skin Innate Immunity and the Skin Microbiome: A Narrative Review. Nutrients. 2021;13(2):302.

2. Zasada M, Budzisz EJ, Alergologii AP, Retinoids. Active molecules influencing skin structure formation in cosmetic and dermatological treatments. Postepy Dermatol Alergol. 2019;36(4):392.

3. Ferreira R, Napoli J, Enver T, Bernardino L, Ferreira L. Advances and challenges in retinoid delivery systems in regenerative and therapeutic medicine. Nat Commun. 2020;11(1):1-14.

4. Torma H. Regulation of keratin expression by retinoids: Dermatoendocrinol 3; 2011. 136-140.

5. Szymański Ł, Skopek R, Palusińska M, Schenk T, Stengel S, Lewicki S, Kraj L, Kamiński P, Zelent A. Retinoic Acid and Its Derivatives in Skin. Cells. 2020;9(12):2660.

6. Varani J, Fligiel H, Zhang J, Aslam MN, Lu Y, Dehne LA, Keller ET. Separation of retinoid-induced epidermal and dermal thickening from skin irritation. Arch Dermatol Res. 2003;295(6):255-62.

7. Kong R, Cui Y, Fisher GJ, Wang X, Chen Y, Schneider LM, Majmudar G. A comparative study of the effects of retinol and retinoic acid on histological, molecular, and clinical properties of human skin. $J$ Cosmet Dermatol. 2016;15(1):49-57. 
8. Emanuele E, Bertona M, Altabas K, Altabas V, Alessandrini G. Anti-inflammatory effects of a topical preparation containing nicotinamide, retinol, and 7-dehydrocholesterol in patients with acne: a gene expression study. Clin Cosmet Investig Dermatol. 2012;5:33.

9. Tang L, Yang X, Liang Y, Xie H, Dai Z, Zheng G. Transcription factor retinoid-related orphan receptor yt: a promising target for the treatment of psoriasis. Front Immunol. 2018;9:1210.

10. Neema S, Mukherjee S, Vasudevan B, Verma R, Moorchung N, Chatterjee M. Vitamin D deficiency after oral retinoid therapy for ichthyosis. Pediatr Dermatol. 2015;32(4):151-5.

11. Galitzer BI. Effect of retinoid pretreatment on outcomes of patients treated by photodynamic therapy for actinic keratosis of the hand and forearm. J Drugs Dermatol. 2011;10(10):1124-32.

12. Sorg 0 , Kuenzli S, Saurat J. Side effects and pitfalls in retinoid therapy: Retinoids and Carotenoids in dermatology. In: Anders V, Madeleine D, editors. Wiley online Library; 2007. p. 225.

13. Kim B-H, Lee Y-S, Kang K-S. The mechanism of retinol-induced irritation and its application to antiirritant development. Toxicol Lett. 2003;146(1):65-73.

14. Fisher GJ, Voorhees JJ. Molecular mechanisms of retinoid actions in skin. FASEB J. 1996;10(9):1002-13.

15. Stratigos AJ, Katsambas AD. The role of topical retinoids in the treatment of photoaging. Drugs. 2005;65(8):1061-72.

16. Saurat JH, Didierjean L, Masgrau E, Piletta PA, Jaconi S, Chatellard-Gruaz D, Gumowski D, Masouye I, Salomon D, Siegenthaler G. Topical retinaldehyde on human skin: biologic effects and tolerance. J Invest Dermatol. 1994;103(6):770-4.

17. Schaefer H, Zesch A. Penetration of vitamin A acid into human skin. Acta Derm Venereol. 1975;74:50-5.

18. Sinico C, Manconi M, Peppi M, Lai F, Valenti D, Fadda AM. Liposomes as carriers for dermal delivery of tretinoin: in vitro evaluation of drug permeation and vesicle-skin interaction. J Control Release. 2005;103(1):123-36.

19. Temova Rakuša Ž, Škufca P, Kristl A, Roškar R. Quality control of retinoids in commercial cosmetic products. J Cosmet Dermatol. 2021;20(4):1166-75.

20. Algahtani MS, Ahmad MZ, Ahmad J. Nanoemulgel for improved topical delivery of retinyl palmitate: formulation design and stability evaluation. Nanomaterials. 2020;10(5):848.

21. Ro J, Kim Y, Kim H, Park K, Lee K-E, Khadka P, Yun G, Park J, Chang ST, Lee J. Pectin micro-and nanocapsules of retinyl palmitate as cosmeceutical carriers for stabilized skin transport. Korean J Physiol Pharmacol. 2015;19(1):59.

22. Castro G, Oliveira C, Mahecha G, Ferreira L. Comedolytic effect and reduced skin irritation of a new formulation of all-trans retinoic acid-loaded solid lipid nanoparticles for topical treatment of acne. Arch Dermatol Res. 2011;303(7):513-20.

23. Lira A, Rossetti FC, Nanclares DM, Neto AF, Bentley MV, Marchetti JM. Preparation and characterization of chitosan-treated alginate microparticles incorporating all-trans retinoic acid. $J$ 
Microencapsul. 2009;26(3):243-50.

24. Waghule T, Singhvi G, Dubey SK, Pandey MM, Gupta G, Singh M, Dua K, Microneedles. A smart approach and increasing potential for transdermal drug delivery system. Biomed Pharmacother. 2019;109:1249-58.

25. McGrath MG, Vucen S, Vrdoljak A, Kelly A, O'Mahony C, Crean AM, Moore A. Production of dissolvable microneedles using an atomised spray process: effect of microneedle composition on skin penetration. Eur J Pharm Biopharm. 2014;86(2):200-11.

26. Modepalli N, Shivakumar HN, McCrudden MT, Donnelly RF, Banga A, Murthy SN. Transdermal delivery of iron using soluble microneedles: dermal kinetics and safety. J Pharm Sci. 2016;105(3):1196-200.

27. Jeong H-R, Kim J-Y, Kim S-N, Park J-H. Local dermal delivery of cyclosporin A, a hydrophobic and high molecular weight drug, using dissolving microneedles. Eur J Pharm Biopharm. 2018;127:23743.

28. Bariya SH, Gohel MC, Mehta TA, Sharma OP. Microneedles: an emerging transdermal drug delivery system. J Pharm Pharmacol. 2012;64(1):11-29.

29. Al-Kasasbeh R, Brady AJ, Courtenay AJ, Larrañeta E, McCrudden MT, O’Kane D, Liggett S, Donnelly RF. Evaluation of the clinical impact of repeat application of hydrogel-forming microneedle array patches. Drug Deliv Transl Res. 2020;10(3):690-705.

30. Hiraishi Y, Nakagawa T, Quan Y-S, Kamiyama F, Hirobe S, Okada N, Nakagawa S. Performance and characteristics evaluation of a sodium hyaluronate-based microneedle patch for a transcutaneous drug delivery system. Int J Pharm. 2013;441(1):570-9.

31. Limcharoen B, Toprangkobsin P, Kröger M, Darvin ME, Sansureerungsikul T, Rujwaree T, Wanichwecharungruang S, Banlunara W, Lademann J, Patzelt A. Microneedle-facilitated intradermal proretinal nanoparticle delivery. J Nanomater. 2020;10(2):368.

32. Hirobe S, Otsuka R, lioka H, Quan Y-S, Kamiyama F, Asada H, Okada N, Nakagawa SJ. Clinical study of a retinoic acid-loaded microneedle patch for seborrheic keratosis or senile lentigo. Life Sci. 2017; 168:24-7.

33. Hiraishi Y, Hirobe S, lioka H, Quan Y-S, Kamiyama F, Asada H, Okada N, Nakagawa SJ. Development of a novel therapeutic approach using a retinoic acid-loaded microneedle patch for seborrheic keratosis treatment and safety study in humans. J Control Res. 2013;171(2):93-103.

34. Pisetpackdeekul P, Supmuang P, Pan-In P, Banlunara W, Limcharoen B, Kokpol C, Wanichwecharungruang S. Proretinal nanoparticles: stability, release, efficacy, and irritation. Int J Nanomedicine. 2016;11:3277.

35. Pukfukdee P, Banlunara W, Rutwaree T, Limcharoen B, Sawutdeechaikul P, Pattarakankul T, Sansureerungsikul T, Toprangkobsin P, Leelahavanichkul A, Panchaprateep R. Solid Composite Material for Delivering Viable Cells into Skin Tissues via Detachable Dissolvable Microneedles. ACS Appl Bio Mater. 2020;3(7):4581-9. 
36. Lee JW, Park J-H, Prausnitz M. Dissolving microneedles for transdermal drug delivery. Biomaterials. 2008;29(13):2113-24.

37. Cheng Z, Lin H, Wang Z, Yang X, Zhang M, Liu X, Wang B, Wu Z, Chen D. Preparation and characterization of dissolving hyaluronic acid composite microneedles loaded micelles for delivery of curcumin. Drug Deliv Transl Res. 2020;10(5):1520-30.

38. Hoffman A. Hydrogels for biomedical applications. Adv Drug Deliv Rev. 2012;64:18-23.

\section{Figures}

A-1

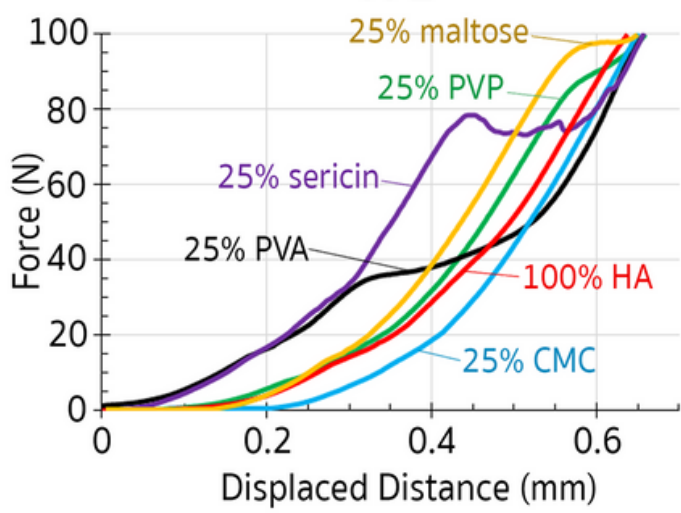

A-2

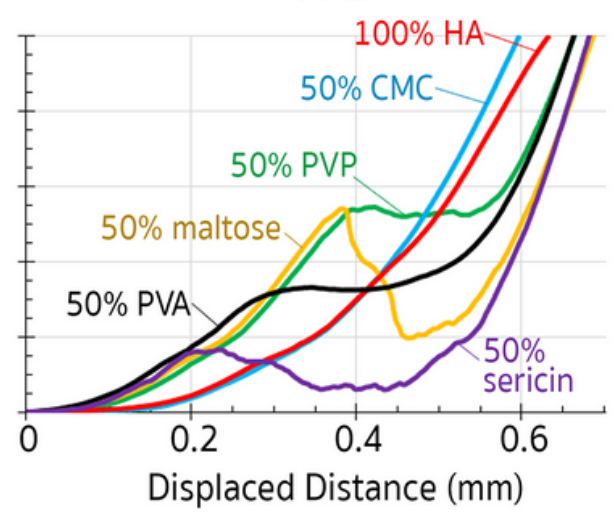

A-3

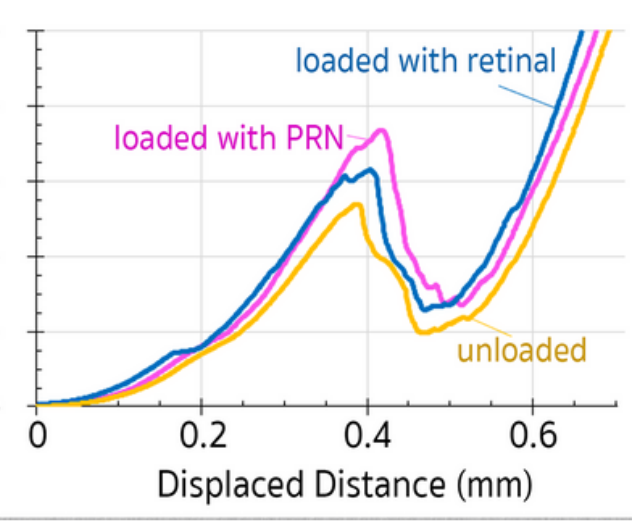

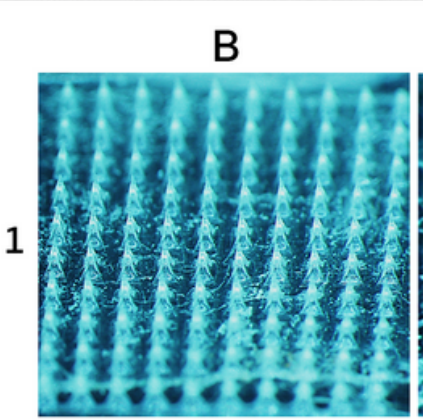
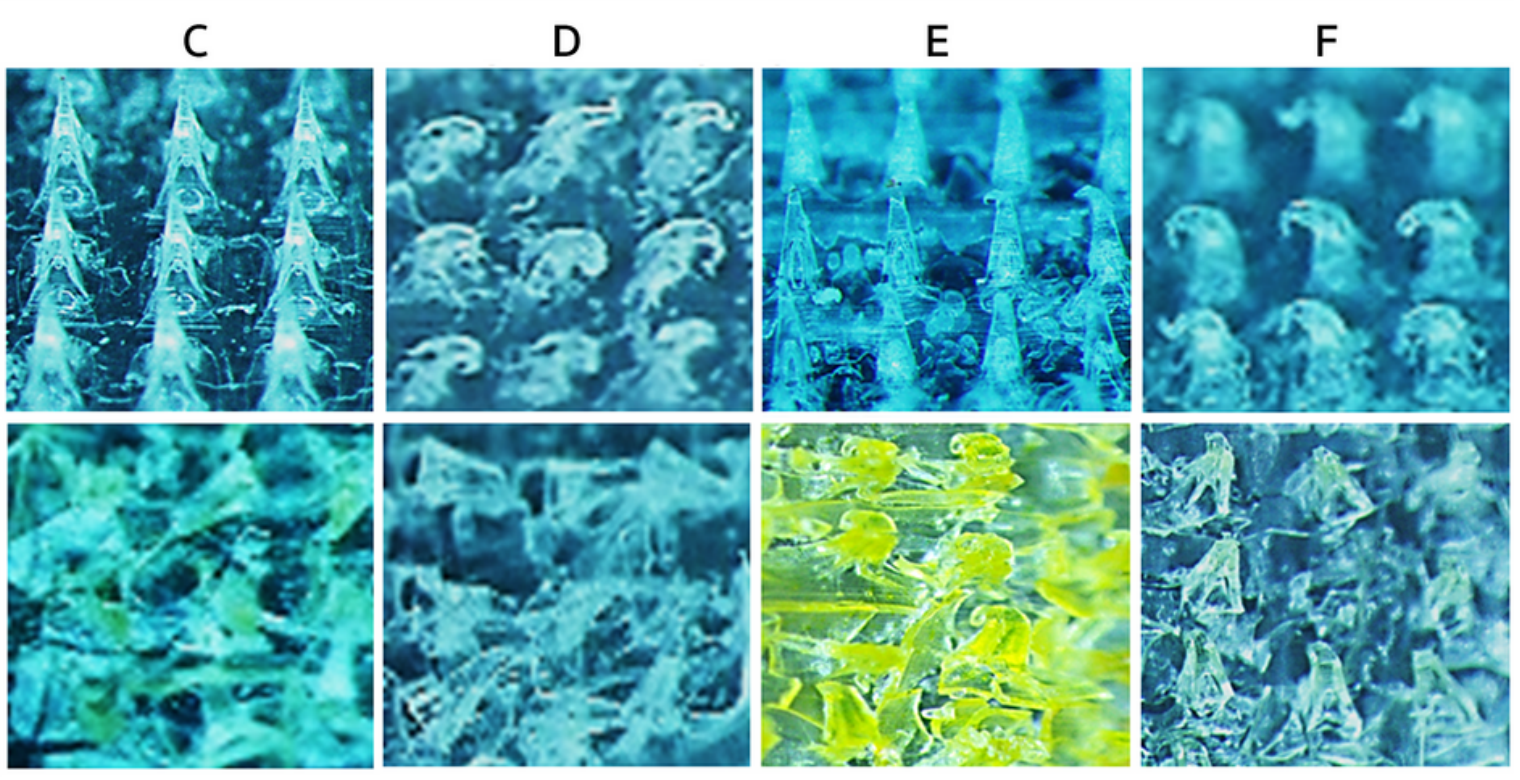

\section{Figure 1}

Mechanical properties (A) and physical appearance (B - F) of DMNs. Compressive strength of DMNs made of Composites of $75 \% \mathrm{HA}$ and $25 \%$ of different second component materials (A-1), Composites of $50 \% \mathrm{HA}$ and $50 \%$ of the second component (A-2), and Composites of 1:1 maltose-HA loaded and not loaded with PRNs or free retinal (A-3). Stereomicroscopic images of DMNs before (B1 and C1) and after (the rest) subjecting to the mechanical testing for DMNs made of $100 \% \mathrm{HA}(\mathrm{D}-1), 50 \% \mathrm{HA}+50 \% \mathrm{PVA}$ (E- 
1), $50 \% \mathrm{HA}+50 \% \mathrm{CMC}(\mathrm{F}-1), 50 \% \mathrm{HA}+50 \% \mathrm{PVP}(\mathrm{B}-2), 50 \% \mathrm{HA}+50 \%$ sericin (C-2), $50 \% \mathrm{HA}+50 \%$ maltose (D-2), 1:1 HA-maltose loaded with PRNs (E-2), 1:1 HA-maltose loaded with retinal (F-2).

A
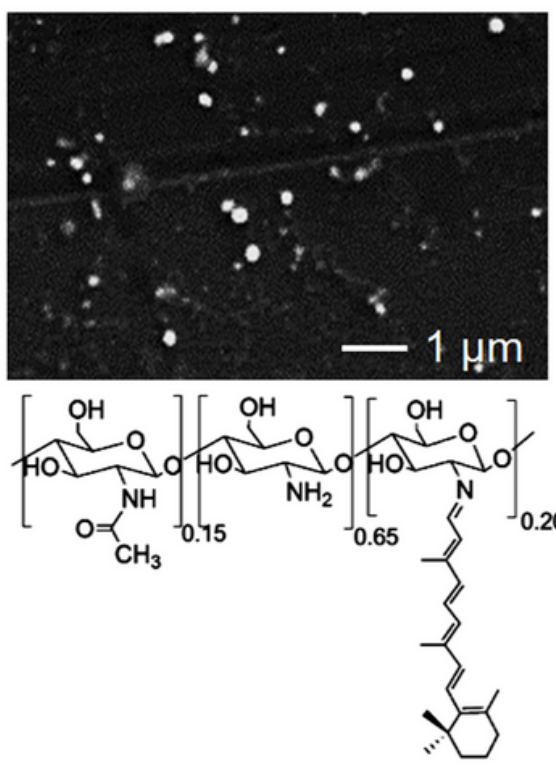

$\mathrm{B}$
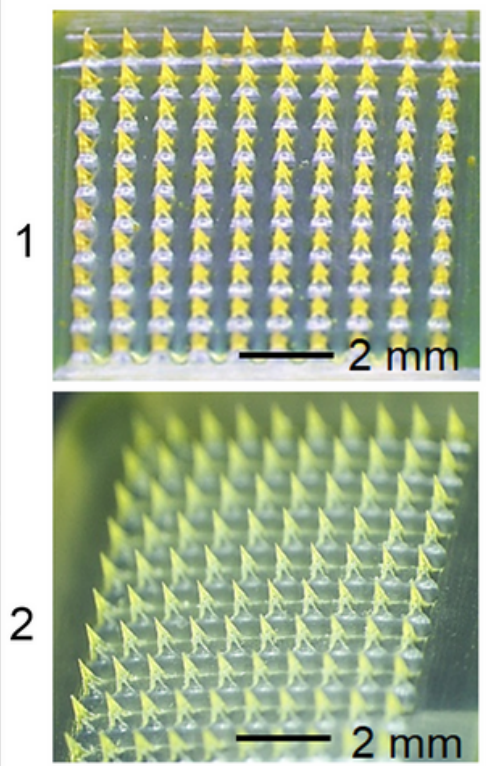

C
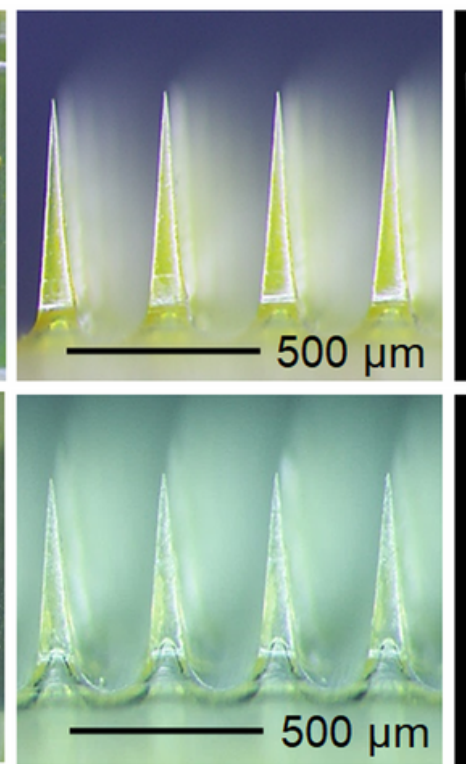
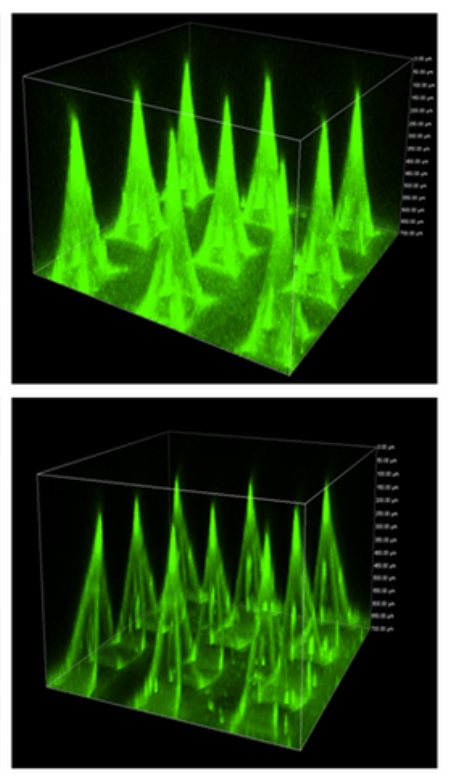

Figure 2

PRN morphology, chemical structure, and the loading into DMNs. SEM image (A top) and chemical structure (A bottom) of PRN particles; Images of PRN-loaded DMNs as taken by stereomicroscope (B1 and $\mathrm{C} 1$ ) and fluorescence microscope (D1); Images of retinal-loaded DMNs as taken by stereomicroscope (B2 and C2) and fluorescence microscope (D2). 

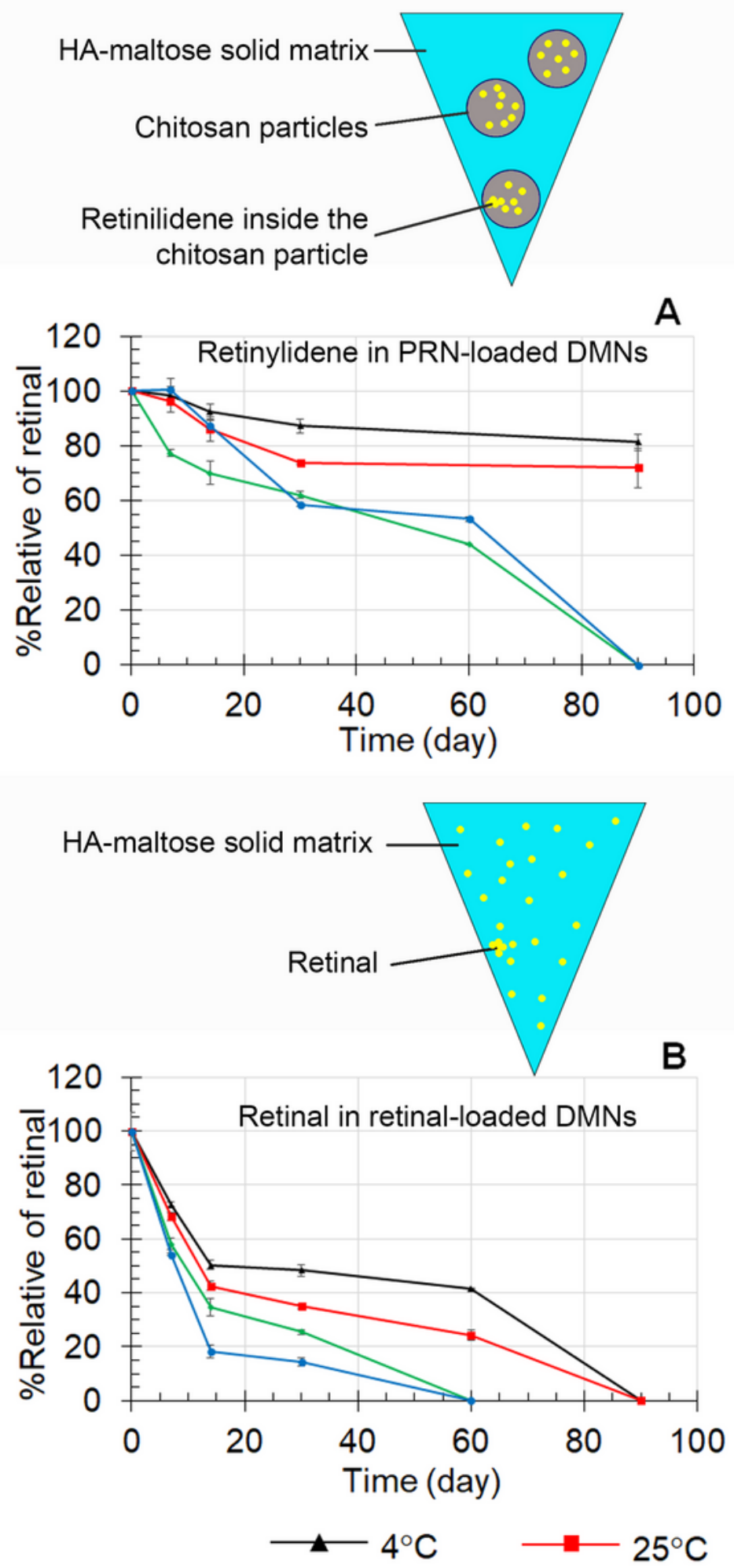
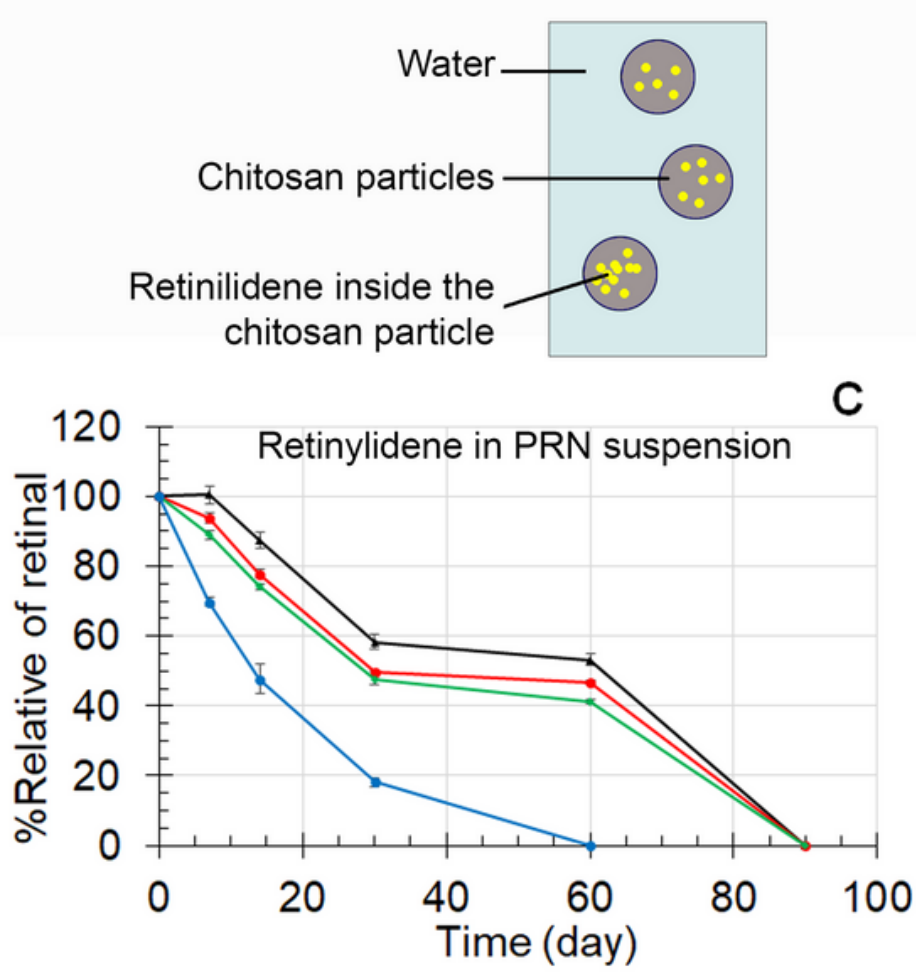

$7 \% \mathrm{v} / \mathrm{v}$ ethanol in water
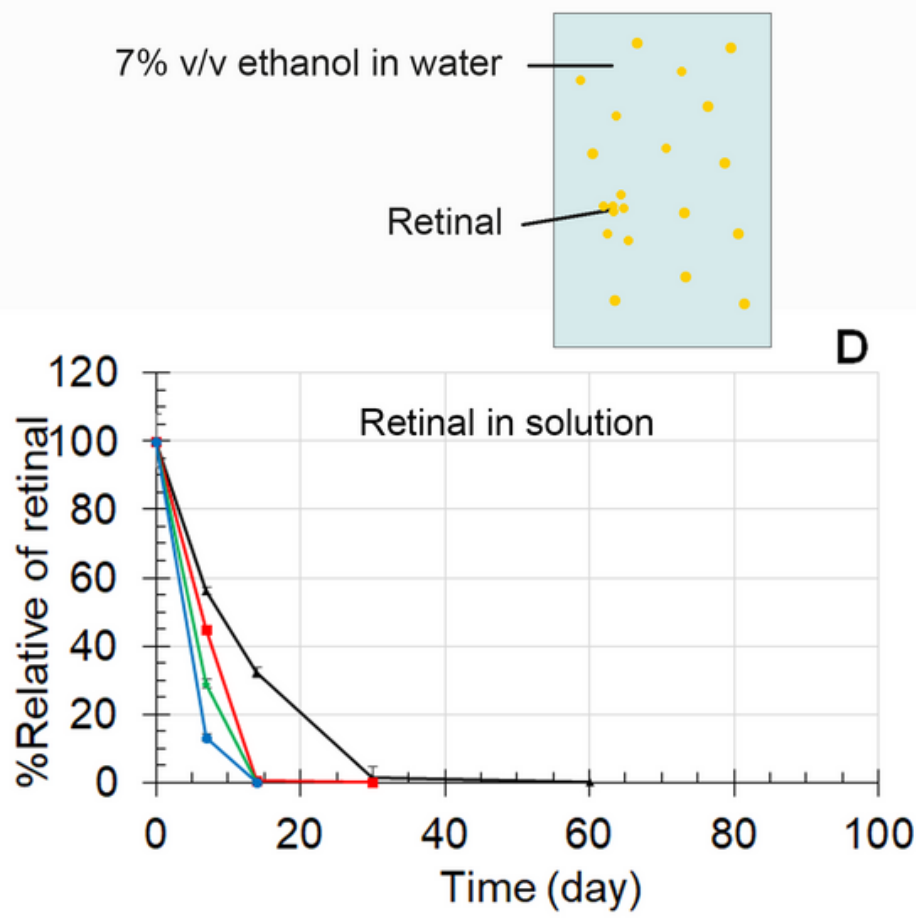

$40^{\circ} \mathrm{C}$

Retinal

Retinal
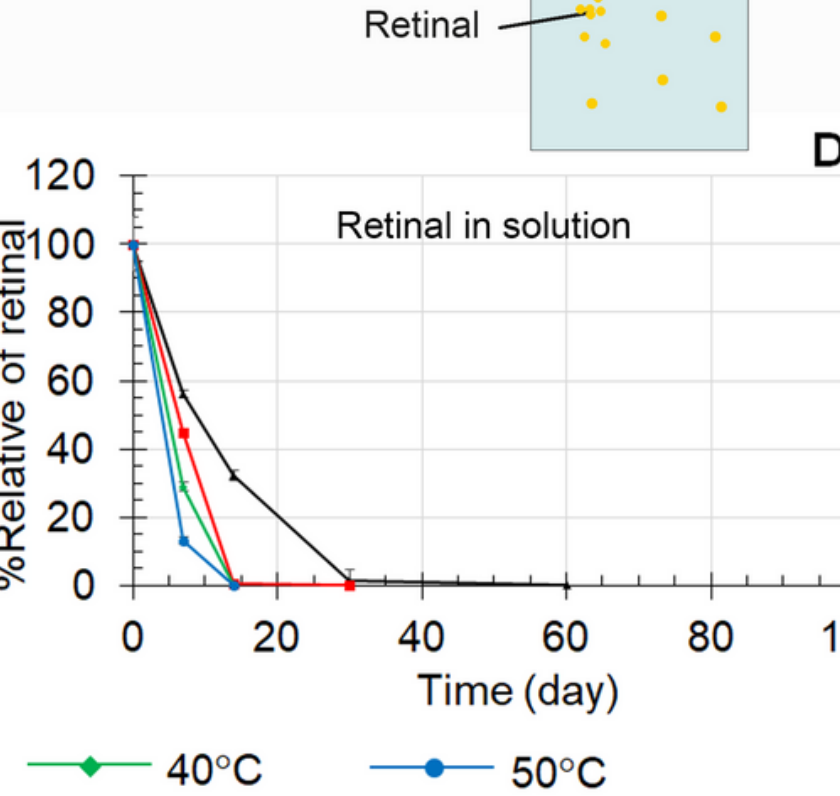

\section{Figure 3}

Plots showing the stability at various temperatures of: Retinylidene moieties in the PRNs when kept in DMNs as PRN-loaded DMNs (A) or as aqueous suspension (B); Retinal when kept in DMNs as retinalloaded DMNs (C) or as retinal solution (D). 


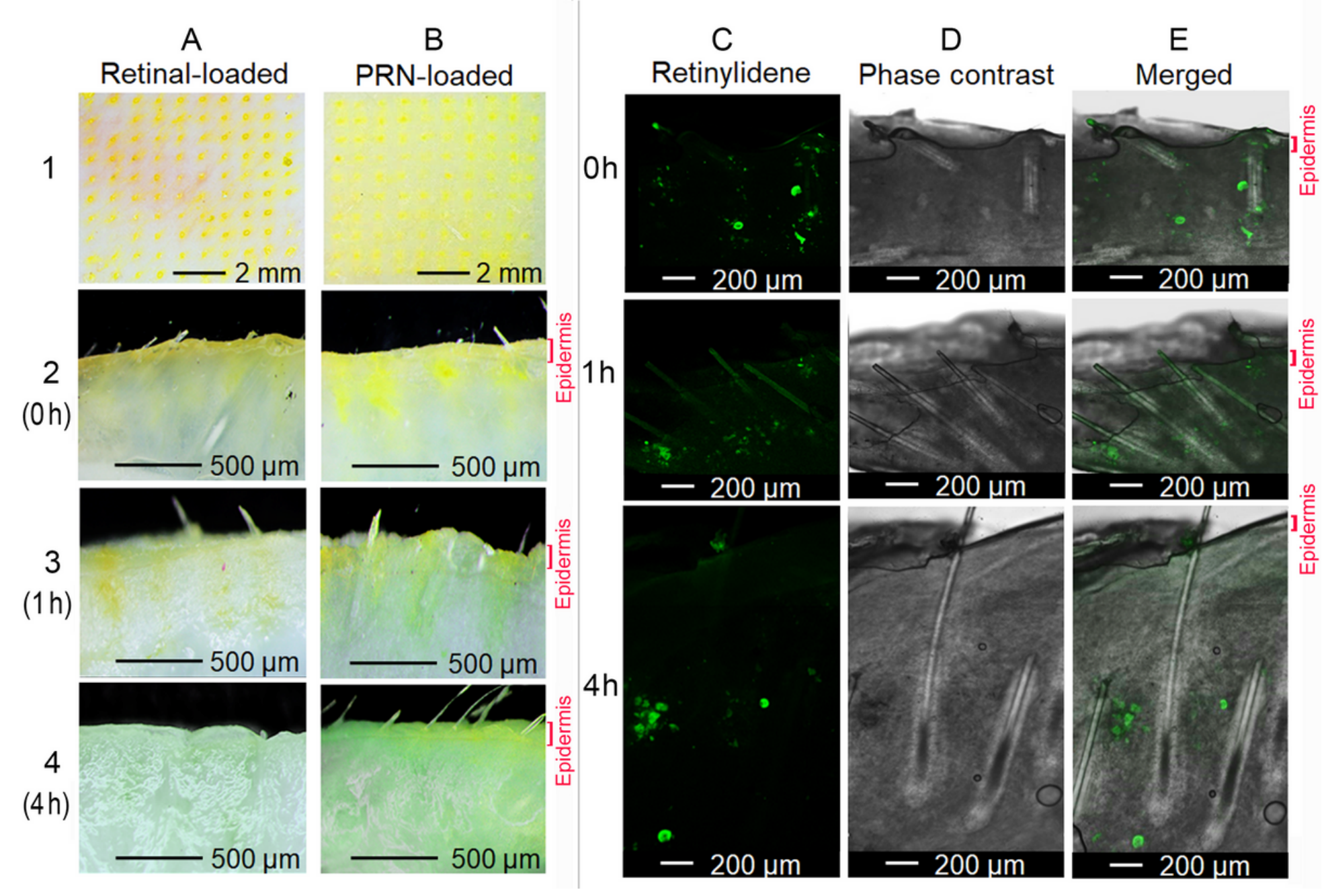

Figure 4

Diffusion of the delivered drug in porcine skin tissue. Stereomicroscopic images of porcine skin after being administered with retinal-loaded DDMNs (column A) or PRN-loaded DDMNs (column B): Top view image of the skin tissue after administration (A1 and B1); Cross sectioned tissue at various times after the administration (A2-A4 and B2-B4). Confocal images of the cross-sectioned porcine skin after being administered with PRN-loaded DDMNs: Fluorescence signal of retinylidene (column C), Phase contrast image (column $\mathrm{D}$ ), and Merged image of column $\mathrm{C}$ and $\mathrm{D}$ (column $\mathrm{E}$ ), at various times post the administration. 


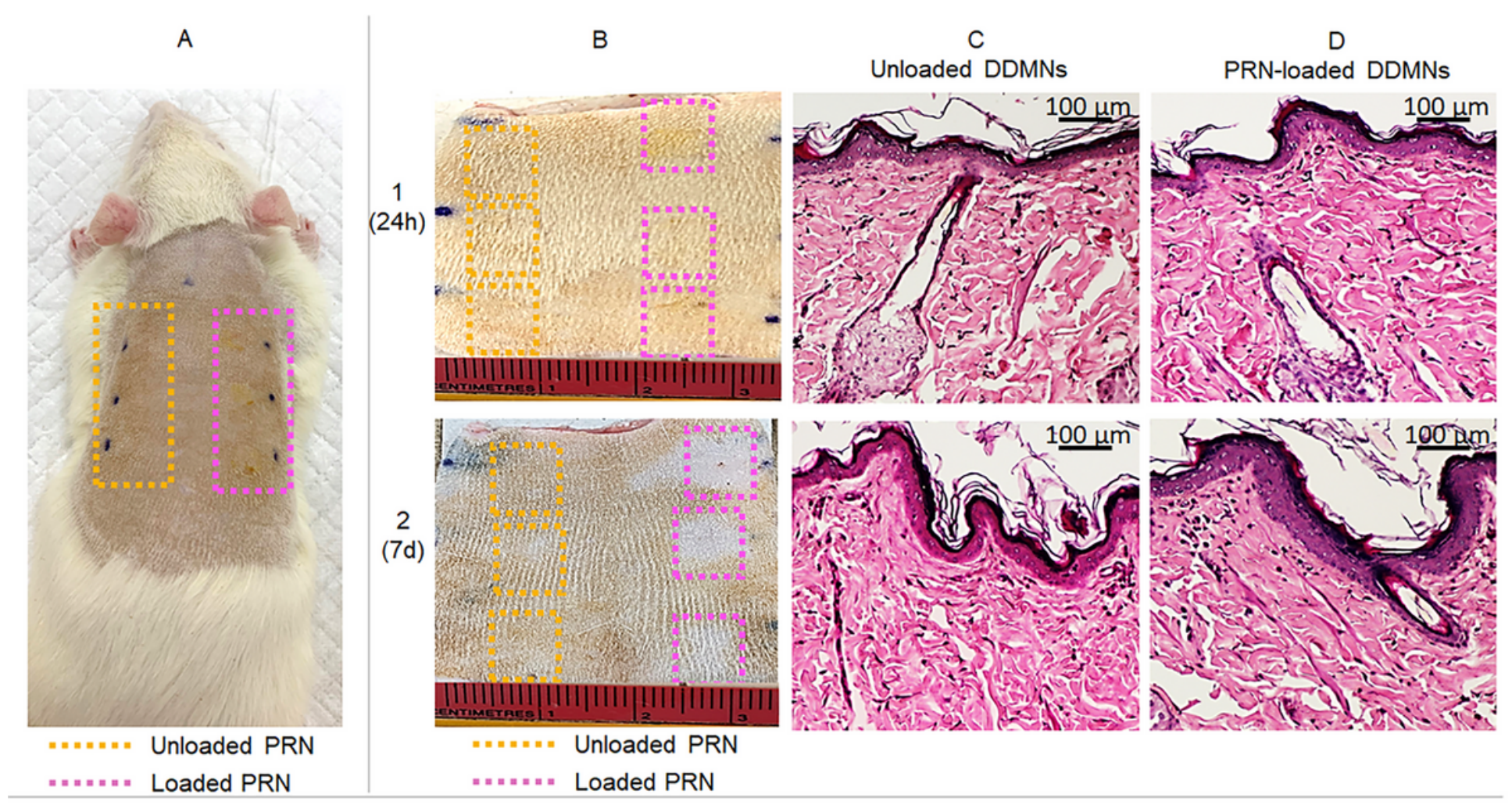

Figure 5

Irritation of rat skin: Representative photographs of rat skin after application of PRN-loaded DDMNs and unloaded DDMNs at $0 \mathrm{~h} \mathrm{(A),} 24 \mathrm{~h}$ (B1) and 7 days (B2) post the administration. Representative images of hematoxylin and eosin-stained cross sectioned histological skin after application of unloaded DDMNs (C1 and C2) and PRN-loaded DDMNs (D1 and D2) at $24 \mathrm{~h}$ (C1 and D1), and 7 days (C2 and D2).

\section{Supplementary Files}

This is a list of supplementary files associated with this preprint. Click to download.

- GraphialAbstract.png 